Article

\title{
Insulation Monitoring Method for DC Systems with Ground Capacitance in Electric Vehicles
}

\author{
Jifei Du ${ }^{1}$, Trillion Q. Zheng ${ }^{1}$, Yian Yan ${ }^{2, *}$, Hongyan Zhao ${ }^{1}$, Yangbin Zeng ${ }^{1}$ and Hong $\mathrm{Li}^{1}$ \\ 1 School of Electrical Engineering, Beijing Jiaotong University, No. 3 Shang Yuan Cun, Haidian District, \\ Beijing 100044, China \\ 2 National Active Distribution Network Technology Research Center (NANTEC), Beijing Jiaotong University, \\ Beijing 100044, China \\ * Correspondence: yanyian@bjtu.edu.cn; Tel.: +86-10-5168-4056; Fax: +86-10-5168-3907
}

Received: 21 May 2019; Accepted: 25 June 2019; Published: 27 June 2019

check for updates

Featured Application: The insulation monitoring generally applied to DC charging piles, battery management systems, or high voltage distribution system for electric vehicles. It is effective on improving the safety performance of electric vehicles.

\begin{abstract}
Owing to the influence of ground capacitance in electric vehicles, in the traditional unbalanced electric bridge DC insulation monitoring (DC-IM) method, the voltage of positive and negative electric bridges changes slowly. To calculate the insulation resistances, sampling should be conducted once the voltage of the bridge becomes stable, that will inevitably extend the monitoring cycle. To reduce the monitoring cycle, this study proposes a three-point climbing algorithm, namely, three-bridge voltage sampling with equal sampling intervals, to predict the evolution of the bridge voltage curve. However, due to the existence of sampling errors, the insulation resistances calculated by sampling values will deviate from the actual values. Then, this article also proposes the filter and correction methods of three sampled voltages to improve monitoring accuracy. Through experimental data, the influences of different parameters on the results are verified, and comparisons with the traditional method are shown in the back. The conclusion is that compared with the traditional method, the proposed method can monitor insulation resistance more quickly and ensure fixed monitoring cycles under different ground capacitance values and keep the similar monitoring accuracy.
\end{abstract}

Keywords: unbalanced electric bridge; DC insulation monitoring; ground capacitance; three-point climbing algorithm

\section{Introduction}

With the increasing popularity of electric vehicles (EVs), strict requirements are being established for the driving and charging safety of EVs. The insulation of EVs decreases due to rain, dampness, collision, and other reasons arising from the long-term exposure of EVs and charging equipment to the outdoor environment [1,2]. The DC system of EVs is connected to numerous power electronic devices, including the motor converter, battery charger, air conditioner, and DC-DC converter [3], and the overall connections may form a DC power microgrid system when EV is charging [4,5]. Insulation failure of any equipment affects the safety of the entire system. When the insulation resistance of the system decreases to below a threshold value, the vehicle sends warning signals. If the situation is serious, then the high-voltage system must be cut off and stopped for troubleshooting [6,7]. The DC insulation monitoring (DC-IM) function is thus required before charging by the DC charging pile and during the process of driving the EVs [8]. Various insulation-monitoring devices and embedded circuits have been designed and installed in DC charging piles, battery packs, high-voltage distribution boxes, 
and other equipment or embedded in the battery management system of EVs. DC-IM methods include balanced electric bridge [9], unbalanced electric bridge [10-14], high-voltage injection [15], differential amplification [16], and low-frequency small-signal injection [17,18]. The unbalanced electric bridge method can synchronously monitor positive and negative insulation resistances, has a low cost, and is easy to realize; thus, it has been widely used in EVs and charging piles.

Because the DC system of EVs connects various power electronic devices that contain many $Y$ capacitors and parasitic capacitors, which make up the large ground capacitance (GC) of the system, GC, an unknown system parameter, seriously affects the monitoring accuracy and speed of DC-IM. Therefore, various solutions have been suggested in the literature. In $[19,20]$, wavelet-transform and chaos theory detection methods were proposed to deal with interference signals. However, these methods are more suitable for a multi-branch DC system with the small-signal injection method than for systems with a large GC. The method based on the Kalman filter and Lyapunov equation proposed in [18] and [21] needs to be recursive step by step. Thus, obtaining the result takes a long time. The traditional sampling and comparison method is frequently used in current practical product applications. After initiating bridge conversion, sampling and calculation are performed only after GC is fully charged so that a stable voltage signal can be sampled. However, this method considerably slows down DC-IM and cannot meet the real-time requirements of EVs and the future development trend of EV safety.

This study proposes a method of unbalanced electric bridge DC-IM based on a three-point climbing algorithm. After switching the bridge, sampling is conducted for three times at equal intervals. The methods of filtering and automatic correction of sampling voltage are also proposed to reduce the result error caused by voltage ripple and sampling resolution. The calculation can predict the voltage value after the completion of GC charging. The method is simple and easy to implement. It does not need to wait for GC charging and multiple sampling, which can considerably increase the detection speed. Thus, the DC-IM period is fixed and unaffected by GC.

The rest of this paper is organized as follows. Section 2 analyzes the unbalanced electric bridge DC-IM with the existing GC. Section 3 proposes the novel method of the three-point climbing algorithm in order to avoid the impact on GC. Section 4 further optimizes the proposed method and describes the implementation method. In Section 5, The experimental data are exhibited to prove the theory. Finally, conclusions are included in Section 6. Some symbols used in the operation optimization are shown in Table 1.

Table 1. List of some symbols used in the operation optimization.

\begin{tabular}{cc}
\hline Symbol & Explanation \\
\hline DC-IM & DC insulation monitoring \\
GC & Ground capacitance \\
$R_{f 1}$ & The negative insulation resistance \\
$R_{f 2}$ & The positive insulation resistance \\
$R_{a}$ & The larger bridge resistance \\
$R_{b}$ & The smaller bridge resistance \\
$C_{1}$ & The capacitance value of the DC negative pole to the earth \\
$C_{2}$ & The capacitance value of the DC positive pole to the earth \\
$v_{1}$ & The voltage value of the DC negative pole to the earth \\
$v_{2}$ & The voltage value of the DC positive pole to the earth \\
$v_{11}$ and $v_{12}$ & The sample value of $v_{1}$ in $M_{1}$ and $M_{2}$ phase respectively \\
$v_{21}$ and $v_{22}$ & The sample value of $v_{2}$ in $M_{1}$ and $M_{2}$ phase respectively \\
$\Delta t$ & The time interval of sampling \\
$E_{1}$ & $e^{-\frac{\Delta t}{\tau_{1}}}$ of $M_{1}$ phase \\
$E_{2}$ & $e^{-\frac{\Delta t}{\tau_{2}}}$ of $M_{2}$ phase \\
$\hat{E}_{1}(k)$ & The estimated value of the $k$ th $E_{1}$ \\
$\hat{E}_{2}(k)$ & The estimated value of the $k$ th $E_{2}$ \\
$\widetilde{v}$ & Estimated voltage value of the half-bridge voltage \\
$\hat{v}$ & Correction voltage value of the half-bridge voltage
\end{tabular}




\section{Traditional Unbalanced Electric Bridge DC-IM Method and Analysis of the Influence on GC}

The unbalanced electric bridge DC-IM topology circuit is shown in Figure 1 , where $v_{\mathrm{dc}}$ is the DC voltage; $R_{f 2}$ and $R_{f 1}$ are the positive and negative insulation resistances, respectively; $R_{a}$ and $R_{b}$ are the bridge resistances, $R_{a}=R_{1}, R_{b}=R_{1} \| R_{2}$, so $R_{a}>R_{b}$. The unbalanced electric bridge method works in two phases, namely, $M_{1}$ and $M_{2}$. In the $M_{1}$ phase, $Q_{1}$ turn-on and $Q_{2}$ turn-off, the positive half-bridge resistance is $R_{a}$, the negative half-bridge resistance is $R_{b}$, the negative and positive half-bridge voltages are $v_{11}$ and $v_{12}$, respectively, and the ground current is $i_{1}$, as shown in Figure $1 \mathrm{a}$. In the $M_{2}$ phase, $Q_{1}$ turn-off and $Q_{2}$ turn-on, the positive half-bridge resistance is $R_{b}$, and the negative half-bridge resistance is $R_{a}$. The negative and positive half-bridge voltages are $v_{21}$ and $v_{22}$, respectively, and the ground current is $i_{2}$, as shown in Figure 1b. The conventional DC-IM method can be expressed as Equation (1) by Kirchhoff's law.

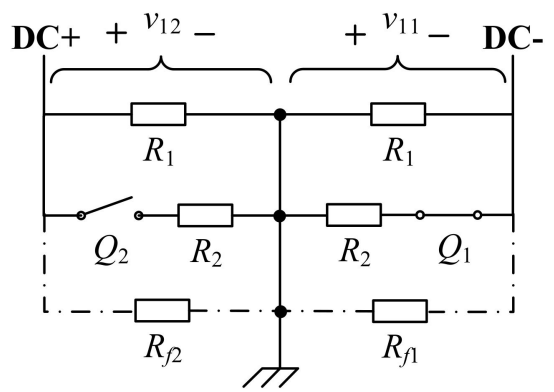

(a)

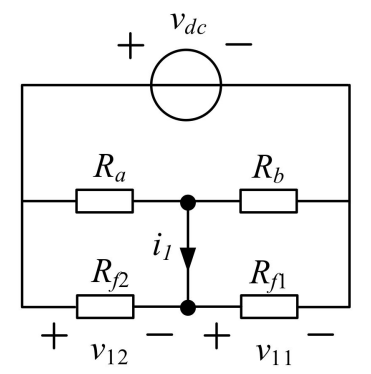

(c)

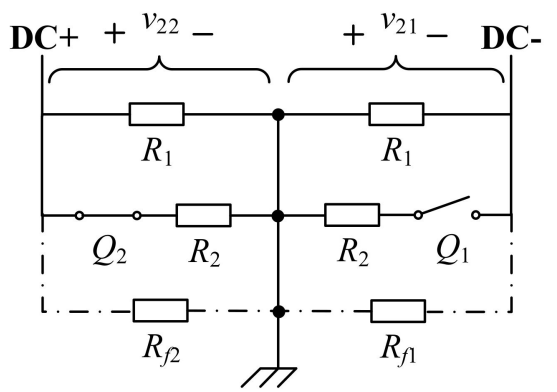

(b)

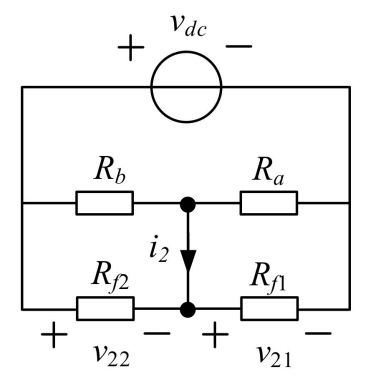

(d)

Figure 1. The circuit of unbalanced bridge. (a) The circuit of $M_{1}$ phase. (b) The circuit of $M_{2}$ phase. (c) The equivalent circuit of $M_{1}$ phase. (d) The equivalent circuit of $M_{2}$ phase.

$$
\left\{\begin{array}{l}
R_{f 1}=\frac{v_{d c}\left(R_{a}-R_{b}\right)-\left(i_{1}-i_{2}\right)\left(R_{a} R_{b}\right)}{i_{1} R_{b}-i_{2} R_{a}} \\
R_{f 2}=\frac{v_{d c}\left(R_{a}-R_{b}\right)-\left(i_{1}-i_{2}\right)\left(R_{a} R_{b}\right)}{i_{1} R_{a}-i_{2} R_{b}}
\end{array}\right.
$$

When the DC system has GC, the capacitance value of the DC negative pole to the earth is $C_{1}$, and the capacitance value of the DC positive pole to the earth is $C_{2}$. Thus, the circuits in Figure $1 \mathrm{c}, \mathrm{d}$ can be changed to those shown in Figure 2a,b. To facilitate calculation and analysis, the equivalent resistance of the two working modes is assumed to be what is shown in Equation (2). Figure 2a,b can be simplified as Figure 2c,d.

$$
\left\{\begin{array}{l}
R_{11}=\frac{R_{b} R_{f 1}}{R_{b}+R_{f 1}}, R_{12}=\frac{R_{a} R_{f 2}}{R_{a}+R_{f 2}} \\
R_{21}=\frac{R_{a} R_{f 1}}{R_{a}+R_{f 1}}, R_{22}=\frac{R_{b} R_{f 2}}{R_{b}+R_{f 2}}
\end{array}\right.
$$




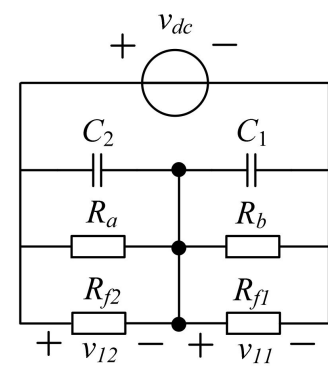

(a)

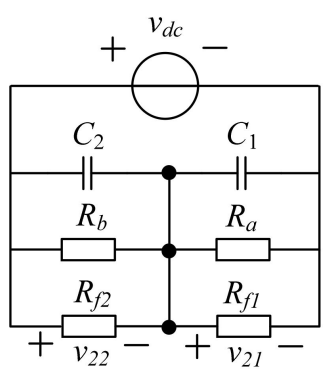

(b)

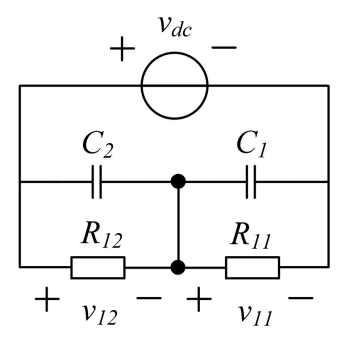

(c)

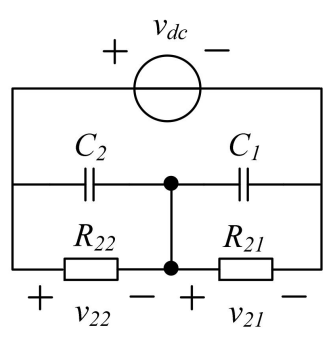

(d)

Figure 2. Equivalent circuit of unbalanced bridge with GC. (a) The circuit of $M_{1}$ phase. (b) The circuit of $M_{2}$ phase. (c) The equivalent circuit of $M_{1}$ phase. (d) The equivalent circuit of $M_{2}$ phase.

The following parameters are set.

$$
\left\{\begin{array}{l}
X_{11}=\frac{R_{11}}{R_{11}+R_{12}}, X_{12}=\frac{R_{12}}{R_{11}+R_{12}} \\
X_{21}=\frac{R_{21}}{R_{21}+R_{22}}, X_{22}=\frac{R_{22}}{R_{21}+R_{22}}
\end{array}\right.
$$

According to Figure 2, the time constants of the $M_{1}$ and $M_{2}$ phases are defined as

$$
\tau_{1}=\frac{\left(C_{1}+C_{2}\right) R_{11} R_{12}}{\left(R_{11}+R_{12}\right)} \text { and } \tau_{2}=\frac{\left(C_{1}+C_{2}\right) R_{21} R_{22}}{\left(R_{21}+R_{22}\right)}
$$

When the two phases switch with each other, the charging process of GC belongs to the first-order circuit full response process, and the curvilinear function Equation (5) can be obtained, where $v_{110}, v_{120}$, $v_{210}$, and $v_{220}$ are the initial voltage of the full response processes of $v_{11}, v_{12}, v_{21}$, and $v_{22}$, respectively.

$$
\left\{\begin{array}{l}
v_{11}=v_{d c} X_{11}+\left(v_{110}-v_{d c} X_{11}\right) e^{-\frac{t}{\tau_{1}}} \\
v_{12}=v_{d c} X_{12}+\left(v_{120}-v_{d c} X_{12}\right) e^{-\frac{t}{\tau_{1}}} \\
v_{21}=v_{d c} X_{21}+\left(v_{210}-v_{d c} X_{21}\right) e^{-\frac{t}{\tau_{2}}} \\
v_{22}=v_{d c} X_{22}+\left(v_{220}-v_{d c} X_{22}\right) e^{-\frac{t}{\tau_{2}}}
\end{array}\right.
$$

\section{New Strategy to Avoid the Impact on GC: Three-Point Climbing Algorithm}

The traditional sampling method when GC exists is shown in Figure 3. In the $M_{1}$ phase, $v_{1}$ decreases slowly, and $v_{2}$ increases slowly. Sampling continues for $v_{1}$ and $v_{2}$ until $t_{11 \_n}$ and $t_{12 \_n}$ respectively; $v_{1}$ falls to stable value $v_{d c} X_{11}$, and $v_{2}$ rises to stable value $v_{d c} X_{12}$. After stabilization, the final $v_{11}$ and $v_{12}$ are obtained. The method switches to the $M_{2}$ phase until $v_{1}$ and $v_{2}$ reach stable values $v_{d c} X_{21}$ and $v_{d c} X_{22}$, respectively, and the controller obtains the final $v_{21}$ and $v_{22}$. One sampling period $T_{C}$ ends, and the insulation resistance values $R_{f 2}$ and $R_{f 1}$ are calculated by $v_{11}=v_{d c} X_{11}, v_{12}$ $=v_{d c} X_{12}, v_{21}=v_{d c} X_{21}$, and $v_{22}=v_{d c} X_{22}$. The resistance values of $R_{11}, R_{12}, R_{21}$, and $R_{22}$ are large, so the charging time of the capacitor is long. Sampling and calculation should be performed after GC charging is completed; hence, the measurement time of the traditional method is long and unable to meet the real-time requirements of EVs. To avoid the measurement overtime caused by GC, a new insulation resistance monitoring method, namely, three-point climbing algorithm, is proposed. 


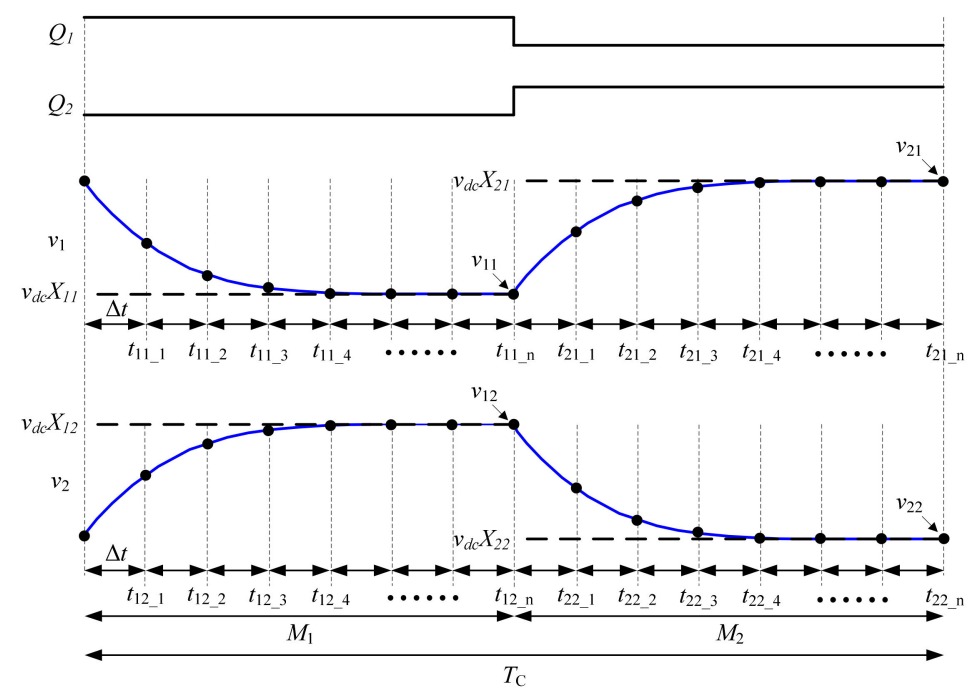

Figure 3. Traditional sampling method.

Figure 4 shows that each phase is sampled three times, and the sampling intervals are equal. With $v_{11}$ as an example, the three sampling times are $t_{11 \_1}, t_{11 \_2}$, and $t_{11 \_3}$; the sampling voltage values are $v_{11 \_}, v_{11} \_$, and $v_{11 \_3}$, respectively, and the time intervals are $\Delta t$. Similar definitions of $v_{12}, v_{21}$, and $v_{22}$ are provided to facilitate the calculation, and $E_{1}$ and $E_{2}$ are used to present natural exponential function. The following equation is then obtained from (5).

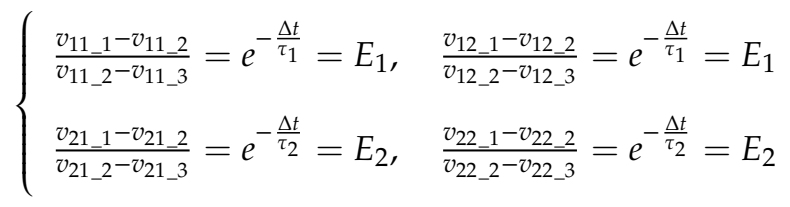

$t_{11 \_1}, t_{12 \_1}, t_{21 \_1}$, and $t_{22 \_1}$ are set as initial times for the first-order circuit full response curve, and the curve Equation (5) is converted into Equation (7).

$$
\left\{\begin{array}{l}
v_{11 \_2}=v_{d c} X_{11}+\left(v_{11 \_1}-v_{d c} X_{11}\right) E_{1} \\
v_{12 \_2}=v_{d c} X_{12}+\left(v_{12 \_1}-v_{d c} X_{12}\right) E_{1} \\
v_{21 \_2}=v_{d c} X_{21}+\left(v_{21 \_1}-v_{d c} X_{21}\right) E_{2} \\
v_{22 \_2}=v_{d c} X_{22}+\left(v_{22 \_1}-v_{d c} X_{22}\right) E_{2}
\end{array}\right.
$$

Equation (7) is converted into Equation (8).

$$
\begin{cases}X_{11}=\frac{v_{11 \_}-v_{11 \_1} E_{1}}{v_{d c}\left(1-E_{1}\right)}, & X_{12}=\frac{v_{12 \_}-v_{12 \_1} E_{1}}{v_{d c}\left(1-E_{1}\right)} \\ X_{21}=\frac{v_{21 \_2}-v_{12 \_1} E_{2}}{v_{d c}\left(1-E_{2}\right)}, & X_{22}=\frac{v_{22 \_\_}-v_{22 \_1} E_{2}}{v_{d c}\left(1-E_{2}\right)}\end{cases}
$$

$X_{11}, X_{12}, X_{21}$, and $X_{22}$ in Equation (8) are known values calculated by sampling voltage. Equation (9) can be obtained from Equations (2), (3), and (8), and insulation resistance values $R_{f 1}$ and $R_{f 2}$ can be solved.

$$
\left\{\begin{array}{l}
R_{f 1}=\frac{R_{a} R_{b}}{\frac{R_{a}-R_{b}}{X_{12}-X_{22}} X_{12}-R_{a}}, R_{f 2}=\frac{R_{a} R_{b}}{\frac{R_{b}-R_{a}}{X_{11}-x_{21}} X_{11}-R_{b}} \\
R_{f 1}=\frac{R_{a} R_{b}}{\frac{R_{a}-R_{b}}{X_{12}-X_{22}} X_{22}-R_{b}}, R_{f 2}=\frac{R_{b}-R_{a} R_{b}}{X_{11}-X_{21}} X_{21}-R_{a}
\end{array}\right.
$$




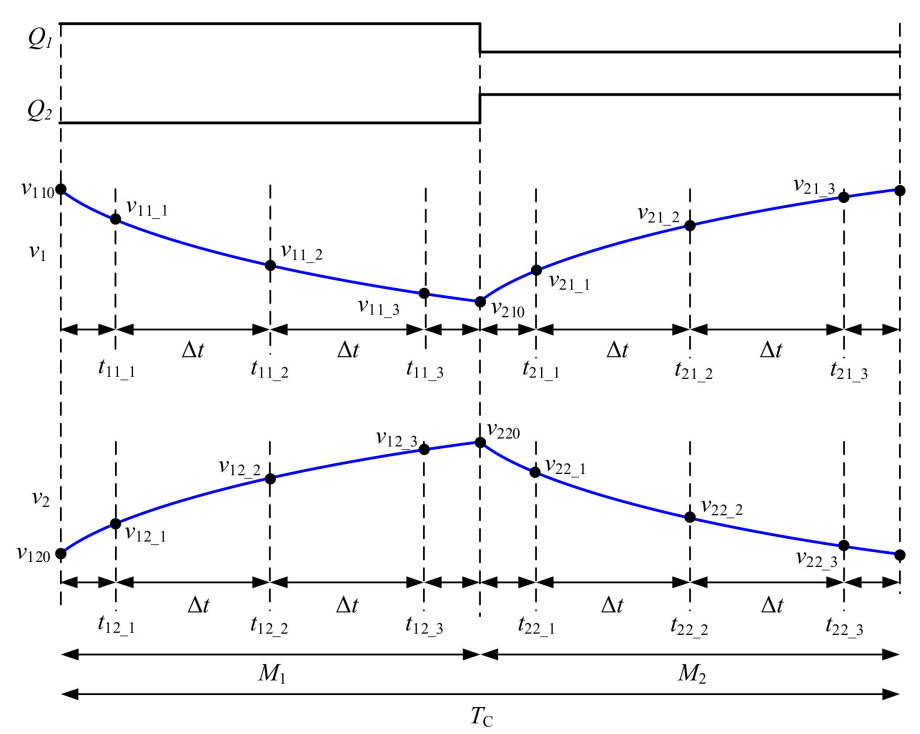

Figure 4. Proposed three-point climbing algorithm.

\section{Implementation Method for Improving Accuracy}

\subsection{Error Analysis}

In practical applications, sample resolution and voltage ripple cause sampling errors, which affect the calculated result of insulation resistance. With the $v_{11}$ of the $M_{1}$ phase as an example, $\Delta v_{11} \_1, \Delta v_{11 \_2}$, and $\Delta v_{11 \_3}$ are the sampling errors of $v_{11 \_1}, v_{11 \_2}$, and $v_{11 \_3}$, respectively. $E_{1 R}$ is the actual value of $E_{1}$. $E_{1 C}$ is the measured value of $E_{1}$. Considering the sampling error, the expression of $E_{1 R}$ and $E_{1 C}$ according to Equation (6) is shown as

$$
\left\{\begin{array}{l}
E_{1 R}=\frac{v_{11 \_2}-v_{11 \_3}}{v_{11} v_{11}-v_{11}^{2}} \\
E_{1 C}=\frac{\left(v_{11 \_2}+\Delta v_{11 \_2}\right)-\left(v_{11 \_3}+\Delta v_{11 \_3}\right)}{\left(v_{11 \_1}+\Delta v_{11 \_1}\right)-\left(v_{11 \_2}+\Delta v_{11 \_2}\right)}
\end{array}\right.
$$

$\Delta E_{1}$ is the error of $E_{1}$ and is defined as follows:

$$
\Delta E_{1}=E_{1 C}-E_{1 R}
$$

By substituting Equation (11) into Equation (10), $\Delta E_{1}$ can be rewritten as

$$
\Delta E_{1}=\frac{\left(\Delta v_{11 \_2}-\Delta v_{11 \_3}\right)-E_{1 R}\left(\Delta v_{11 \_1}-\Delta v_{11 \_2}\right)}{\left(v_{11 \_1}-v_{11 \_2}\right)+\left(\Delta v_{11 \_1}-\Delta v_{11 \_2}\right)}
$$

where $\Delta v_{11 \_1}, \Delta v_{11 \_2}$, and $\Delta v_{11 \_3}$ are uncontrollable components and $E_{1 R}$ is a fixed value. $\left(v_{11 \_1} v_{11 \_2}\right)$ is inversely proportional to $\Delta E_{1}$. Similarly, $v_{12}, v_{21}$, and $v_{22}$ can result in the same conclusion. In the application, the larger $\Delta t_{11}, \Delta t_{12}, \Delta t_{21}$, and $\Delta t_{22}$ are, the smaller the result error is. The larger the difference between the $R_{a}$ and $R_{b}$ is, the smaller the result error is. The higher the $v_{\mathrm{dc}}$ is, the smaller the result error is.

\subsection{Selection of Calculation Method}

In the climbing stage, the smaller GC is, the larger the difference is in the three-point sampling and the smaller the result error is. If GC is too small, the sampling may reach the stable stage after climbing, the difference between the three sampled voltages will be too small, and the resulting error will increase. The accuracy is the highest only when the three sampled voltages are in the climbing stage of the voltage variation curve. Equation (6) shows that when the difference of the three sampled voltages is close to zero, the calculated value of $\Delta E_{1}$ is nearly infinite because of signal interference in 
the actual application unlike in the ideal situation. This condition seriously affects the measurement result. A positive constant is set as $C_{a}$ to determine if GC is too small by using the following rule.

$$
\left\{\begin{array}{l}
v_{11 \_2}-v_{11 \_3}<\mathrm{C}_{\mathrm{a}} \\
v_{22 \_2}-v_{22 \_3}<\mathrm{C}_{\mathrm{a}} \\
v_{12 \_2}-v_{12 \_3}>-\mathrm{C}_{\mathrm{a}} \\
v_{21 \_2}-v_{21 \_3}>-\mathrm{C}_{\mathrm{a}}
\end{array}\right.
$$

If the three sampled voltages satisfy Equation (13), then GC is too small to be non-negligible, and the traditional sampling method is adopted. If the three sampled voltages do not satisfy Equation (13), then GC is non-negligible, and the proposed three-point climbing algorithm is adopted.

\subsection{Filter of $E_{1}$ and $E_{2}$}

$\Delta t$ is an invariant constant; $\tau_{1}$ and $\tau_{2}$ vary with GC, so $E_{1}$ and $E_{2}$ are also variations. Counter $k$ is increased once every measurement period $T_{C}$, as shown in Figure 5 . To make $k$ th measurements $E_{1}$ and $E_{2}$ close to the actual value, the average value of $M_{1}$ and $M_{2}$ phases are taken according to Equation (6). $E_{1}(k)$ and $E_{2}(k)$ can be rewritten as

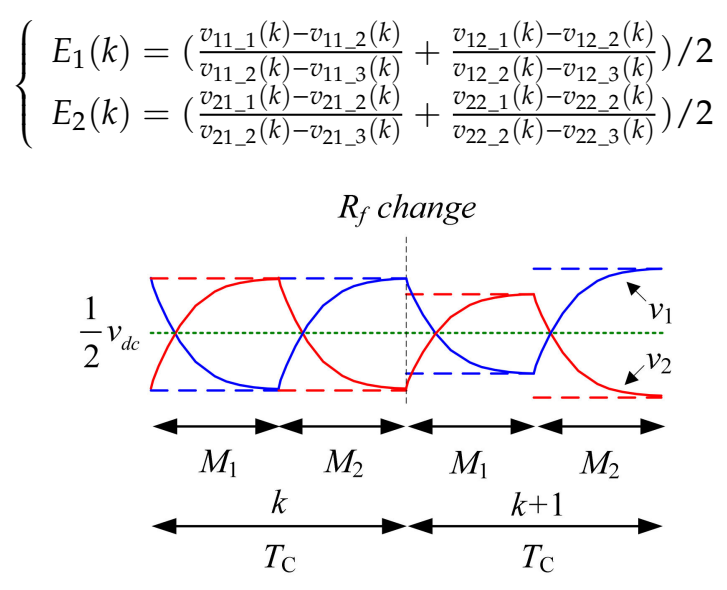

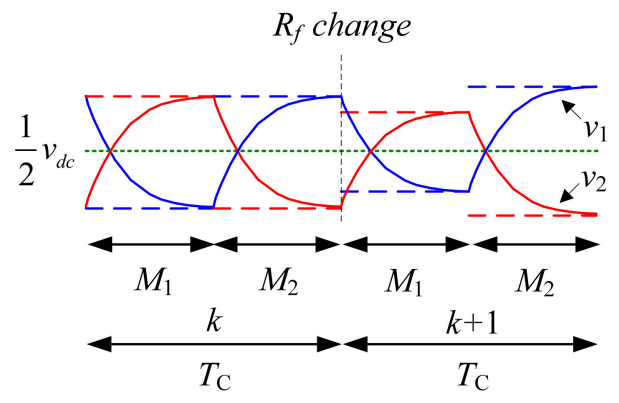

Figure 5. Waveform of the bridge voltage.

The estimated value of the $k$ th $E_{1}$ and $E_{2}$ is set as $\hat{E}_{1}(k)$ and $\hat{E}_{2}(k)$, respectively, which can be obtained by the following first-order filter, where $\mathrm{A}$ is a filter coefficient that satisfies $0<\mathrm{A}<1$.

$$
\left\{\begin{array}{l}
\hat{E}_{1}(k)=\mathrm{A} \hat{E}_{1}(k-1)+(1-\mathrm{A}) E_{1}(k) \\
\hat{E}_{2}(k)=\mathrm{A} \hat{E}_{2}(k-1)+(1-\mathrm{A}) E_{2}(k)
\end{array}\right.
$$

\subsection{Correction of Sampled Value}

The actual sampled voltage value shows a certain deviation from the expected value. The red sampled point in Figure 6 shows that the exponential function curve cannot be formed. The result calculated by the sampled value must be a large error. Therefore, to obtain satisfactory results, the sampled values must be corrected with a loop iterative correction method. $\hat{v}_{11 \_1}(i), \hat{v}_{11 \_2}(i), \hat{v}_{11 \_3}(i), \hat{v}_{12 \_}(i), \hat{v}_{12 \_}(i), \hat{v}_{12 \_3}(i), \hat{v}_{21 \_1}(i), \hat{v}_{21 \_2}(i), \hat{v}_{21 \_}(i), \hat{v}_{22 \_}(i), \hat{v}_{22 \_} 2(i)$, and $\widetilde{v}_{22 \_3}(i)$ are set as the $i$ th correction voltage values. After the 12 voltages of $v_{11}, v_{12}, v_{21}$, and $v_{22}$ are sampled completely, the counter is set as $i=0$. The 12 voltage values are substituted into the following equation, and the sampled value is used as the initial correction value.

$$
\left\{\begin{array}{l}
\hat{v}_{11 \_1}(0)=v_{11 \_1} \\
\hat{v}_{11 \_2}(0)=v_{11 \_2} 2 \\
\hat{v}_{11 \_3}(0)=v_{11 \_3}
\end{array},\left\{\begin{array} { l } 
{ \hat { v } _ { 1 2 \_ 1 } ( 0 ) = v _ { 1 2 \_ 1 } } \\
{ \hat { v } _ { 1 2 \_ 2 } ( 0 ) = v _ { 1 2 \_ } 2 } \\
{ \hat { v } _ { 1 2 \_ 3 } ( 0 ) = v _ { 1 2 \_ 3 } }
\end{array} \left\{\begin{array}{l}
\hat{v}_{21 \_1}(0)=v_{21 \_1} \\
\hat{v}_{21 \_2}(0)=v_{21 \_2} \\
\hat{v}_{21 \_3}(0)=v_{21 \_3}
\end{array},\left\{\begin{array}{l}
\hat{v}_{22 \_1}(0)=v_{22 \_1} \\
\hat{v}_{22 \_2}(0)=v_{22 \_2} \\
\hat{v}_{22 \_3}(0)=v_{22 \_3}
\end{array}\right.\right.\right.\right.
$$




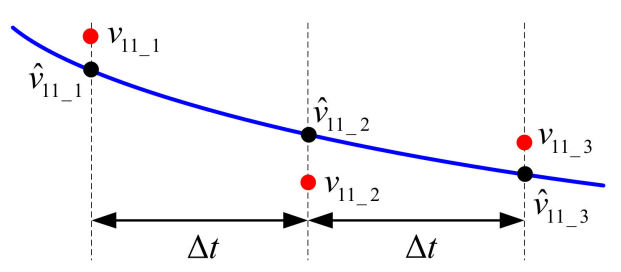

Figure 6. Actual sampling point.

The three sampled voltages of each group cannot form an exponential curve of $\hat{E}_{1}(k)$ due to the measurement error and ripple. To form the desired exponential curve, each voltage value can be estimated by the two other voltage values. $\widetilde{v}_{11 \_1}(i), \widetilde{v}_{11 \_2}(i), \widetilde{v}_{11 \_3}(i), \widetilde{v}_{12 \_1}(i), \widetilde{v}_{12 \_2}(i), \widetilde{v}_{12 \_3}(i), \widetilde{v}_{21 \_1}(i), \widetilde{v}_{21 \_2}(i), \widetilde{v}_{21 \_3}(i), \widetilde{v}_{22 \_1}(i), \widetilde{v}_{22 \_}(i)$, and $\widetilde{v}_{22 \_3}(i)$ are set as the estimated voltage values. The estimated method can be derived from the following equation according to Equation (6).

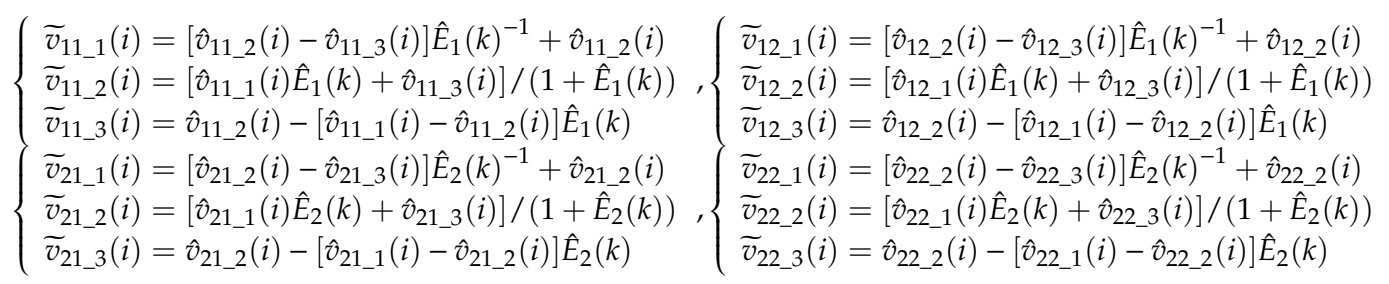

The estimated value comparison rule is shown as

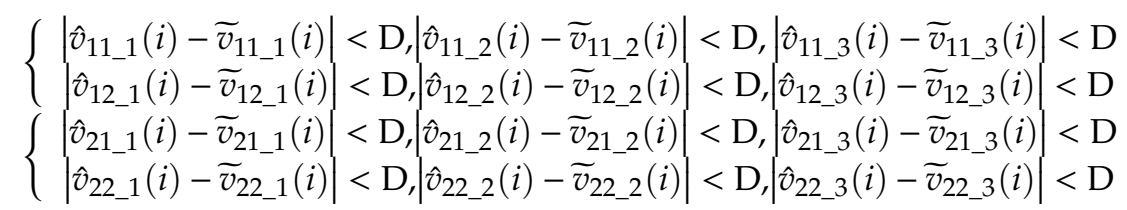

When Equation (18) is satisfied, the difference between the estimated value and the correction value is small, and the $i$ th correction value is applied as the final correction value. Otherwise, the counter $i$ is increased by 1 , and further correction is be conducted as follows:

$$
\begin{aligned}
& \left\{\begin{array}{l}
\hat{v}_{11 \_1}(i+1)=\widetilde{v}_{11 \_1}(i)+\mathrm{B}\left[\hat{v}_{11 \_1}(i)-\widetilde{v}_{11 \_1}(i)\right] \\
\hat{v}_{11 \_2}(i+1)=\widetilde{v}_{11}(i)+\mathrm{B}\left[\hat{v}_{11 \_2}(i)-\widetilde{v}_{11}(i)\right] \\
\hat{v}_{11 \_3}(i+1)=\widetilde{v}_{11 \_3}(i)+\mathrm{B}\left[\hat{v}_{11 \_3}(i)-\widetilde{v}_{11 \_3}(i)\right]
\end{array},\left\{\begin{array}{l}
\hat{v}_{12 \_1}(i+1)=\widetilde{v}_{12 \_1}(i)+\mathrm{B}\left[\hat{v}_{12 \_1}(i)-\widetilde{v}_{12 \_1}(i)\right] \\
\hat{v}_{12 \_2}(i+1)=\widetilde{v}_{12 \_}(i)+\mathrm{B}\left[\hat{v}_{11 \_}(i)-\widetilde{v}_{12 \_}(i)\right] \\
\hat{v}_{12 \_3}(i+1)=\widetilde{v}_{12 \_3}(i)+\mathrm{B}\left[\hat{v}_{12 \_3}(i)-\widetilde{v}_{12 \_3}(i)\right]
\end{array}\right.\right.
\end{aligned}
$$

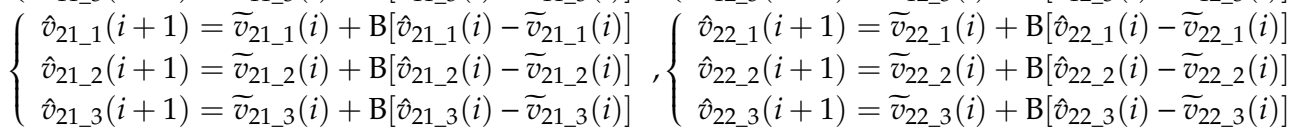

where $B$ is the correction factor that satisfies $0<B<1$. Then, the results of Equation (19) are substituted into Equation (17). This method cycles back and forth until the difference between the estimated and correction values satisfies Equation (18). The cycle is then stopped, and the final correction value is outputted.

The overall software flow chart of the method is shown in Figure 7. 


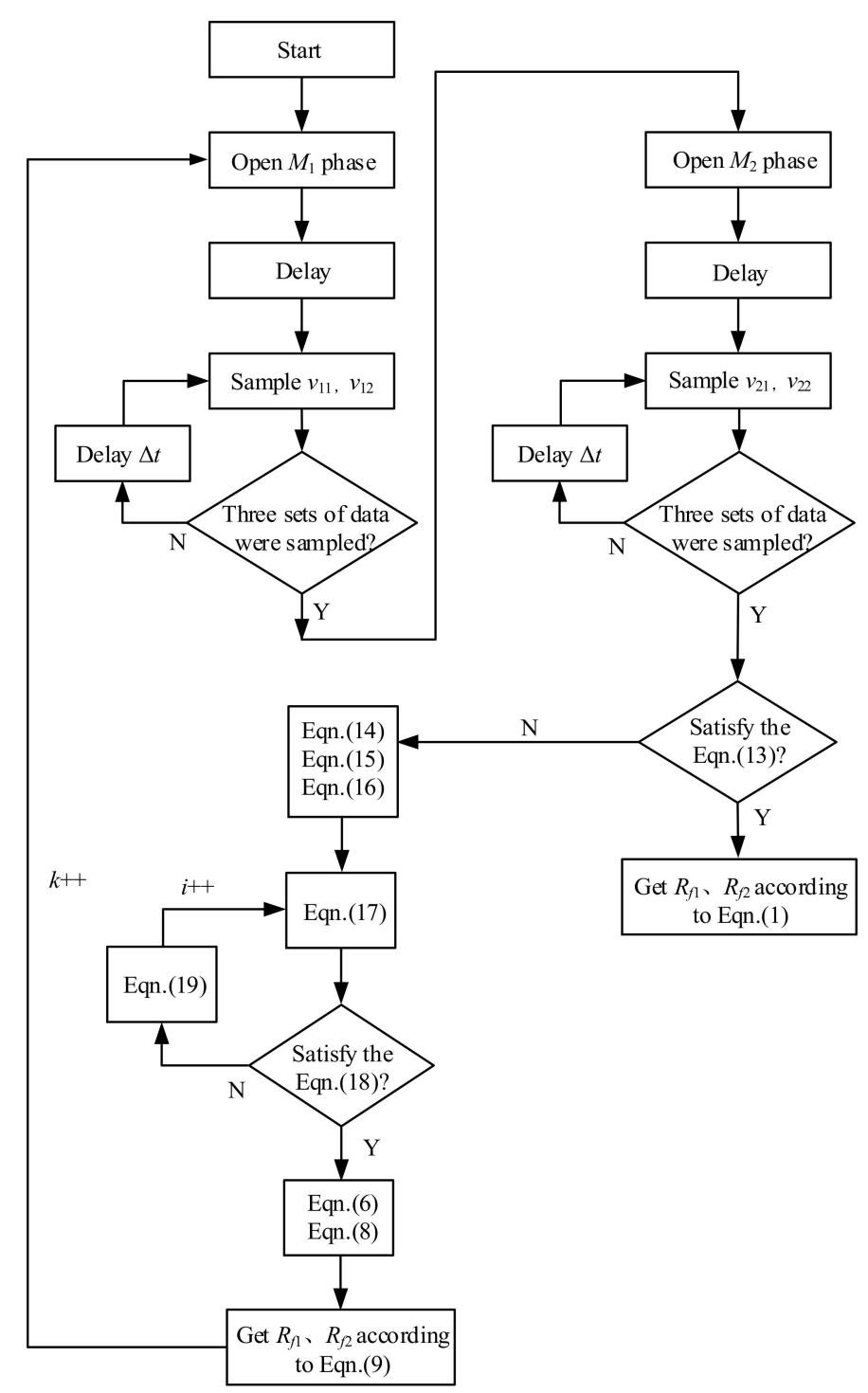

Figure 7. Software flow chart of the proposed method.

\section{Comparative Study of Experimental Data}

The DC-IM device with the unbalanced electric bridge method is created, and the schematic overview of application is shown in Figure 8. The MCU performs the switch $Q_{1}$ and $Q_{2}$, sample the voltage values of $v_{1}$ and $v_{2}$ stored in memory, then calculate the $R_{f 1}$ and $R_{f 2}$, and output the result to the computer. The experiment table is shown in Figure 9. It includes the display interface, DC-IM device, voltage regulating device, insulation resistance selection switch, and GC selection switch. The DC-IM controller uses a PIC18F4580 single-chip microcomputer. The monitoring period is $0.2 \mathrm{~s}$; that is, the switch action occurs every $0.1 \mathrm{~s}$, so the sampling time should satisfy $\left(t_{1}+2 \Delta t\right)<0.1 \mathrm{~s}$. The positive grounding resistance is $R_{f 1}=1000 \mathrm{k} \Omega$, the negative grounding resistance is $R_{f 2}=300 \mathrm{k} \Omega$, the first sampling time $t_{1}=0.01 \mathrm{~s}$, the bridge resistance is $R_{a}=1000 \mathrm{k} \Omega$, and $R_{b}=200 \mathrm{k} \Omega$. The GC value is $C_{Y}=C_{1}=C_{2}$. The grounding current waveform corresponding to different GCs is shown in Figure 10. The waveform of $C_{Y}=0.1 \mu \mathrm{F}$ can be stabilized in a half cycle. The larger the value of $C_{Y}$ is, the closer the waveform is to the triangle wave. Therefore, the traditional unbalanced bridge sampling method is used when $C_{Y}<0.1 \mu \mathrm{F}$, and the three-point climbing algorithm is used when $C_{Y}>0.1 \mu \mathrm{F}$.

The calculation results are compared by changing the different parameters, and relative error $(\mathrm{RE} \%)$ is determined as 


$$
\mathrm{RE} \%=\mid \text { measured value }- \text { actual value } \mid / \text { measured value. }
$$

Different parameters are applied in the proposed method. (1) DC voltage $v_{\mathrm{dc}}=800 \mathrm{~V}, \mathrm{GC}$ value $C_{Y}=0.1 \mu \mathrm{F}$, and the sampling time interval $\Delta t$ is changed; the results are shown in Figure 11. The larger the sampling time interval is, the higher accuracy is. (2) DC voltage $v_{\mathrm{dc}}=800 \mathrm{~V}$, sampling time interval $\Delta t=0.04 \mathrm{~s}$, and the value of $C_{Y}$ is changed; the results are shown in Figure 12. The smaller $C_{Y}$ is, the higher accuracy is. (3) Sampling interval $\Delta t=0.04 \mathrm{~s}, C_{\mathrm{Y}}=0.1 \mu \mathrm{F}$, and DC voltage $v_{\mathrm{dc}}$ is changed; the results are shown in Figure 13. The larger the DC voltage is, the higher accuracy is. When the DC voltage drops to below $200 \mathrm{~V}$, the measurement accuracy is greatly reduced. (4) Under the premise that the parallel value of bridge resistance $R_{a} \| R_{b}$ is constant and the difference between $R_{a}$ and $R_{b}$ is changed; the results are shown in Table 2. The larger the difference between $R_{a}$ and $R_{b}$ is, the higher accuracy is. Time interval $\Delta t$ increases, $C_{\mathrm{Y}}$ decreases, DC voltage $v_{\mathrm{dc}}$ increases, and the difference between $R_{a}$ and $R_{b}$ increases. These factors make the sampled voltage difference larger, which will reduce the error of the final results.

The proposed method is compared with the traditional method to verify the availability and superiority of the former. The monitoring time and relative error of the two methods are shown in Tables 3-5. The relative error is the larger one between $R_{f 1}$ and $R_{f 2}$. Table 3 is the data at $v_{\mathrm{dc}}=800 \mathrm{~V}$, $R_{f 1}=1000 \mathrm{k} \Omega, R_{f 2}=300 \mathrm{k} \Omega$; Table 4 is the data at $v_{\mathrm{dc}}=400 \mathrm{~V}, R_{f 1}=1000 \mathrm{k} \Omega, R_{f 2}=300 \mathrm{k} \Omega$; Table 5 is the data at $v_{\mathrm{dc}}=800 \mathrm{~V}, R_{f 1}=100 \mathrm{k} \Omega, R_{f 2}=100 \mathrm{k} \Omega$. The traditional method needs to increase the monitoring time with a large value of GC because it should have a stable sample value after charging the GC. The proposed method has a fixed monitoring time due to the fixed three-point sampling. When GC is large and the proposed method is applied, the error is similar to using the traditional method. When GC is small, such as $10 \mathrm{nF}$ in the table, and the traditional unbalanced bridge calculation method is applied automatically, the calculation results of two methods are almost the same. Overall, the experimental results are consistent with the theoretical conclusion.

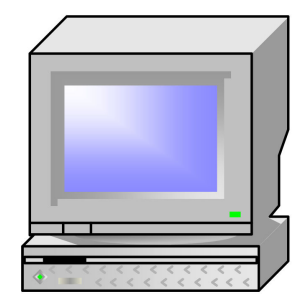

upper computer

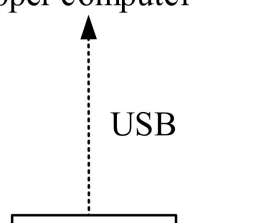

converter

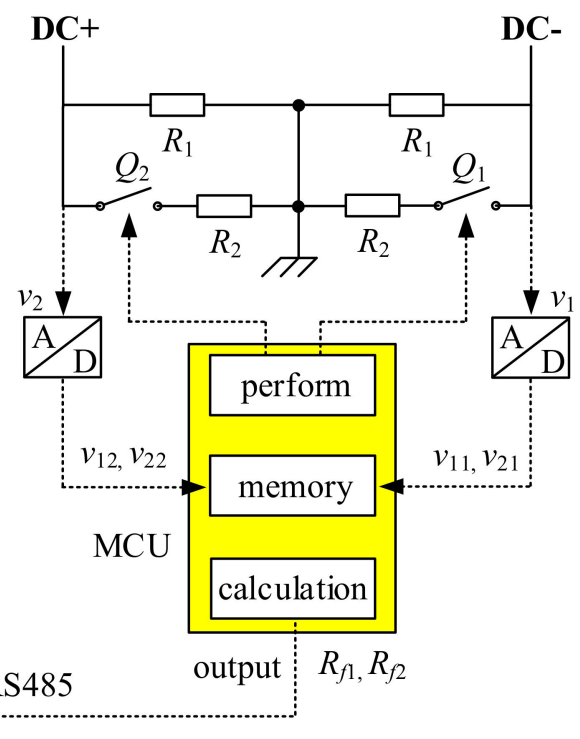

Figure 8. The schematic overview of application. 


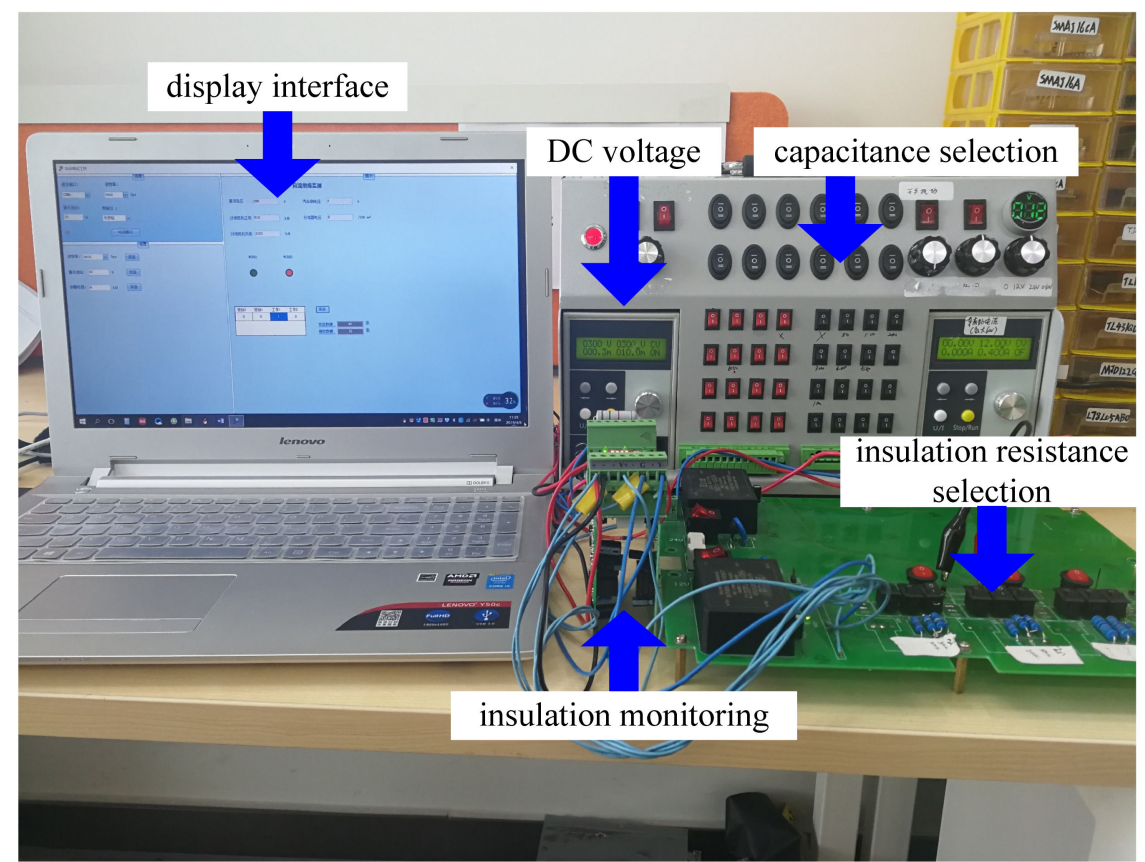

Figure 9. Display of the experiment table.

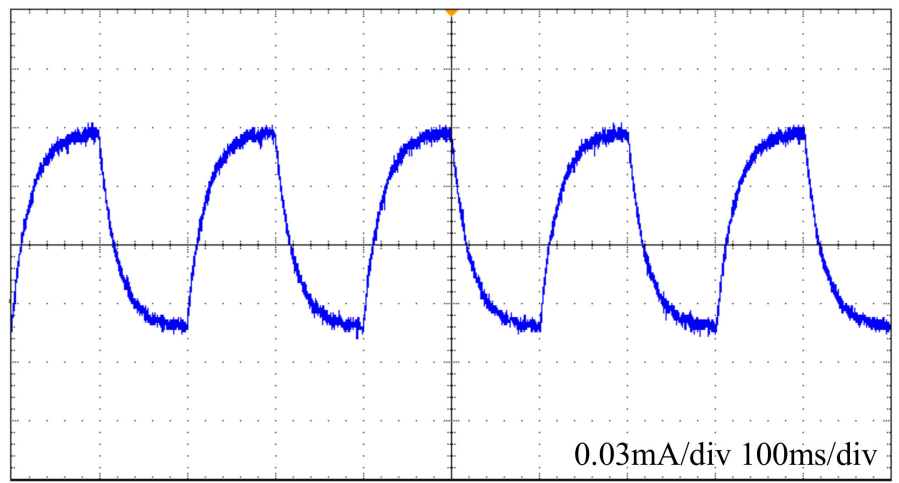

(a) $C_{Y}=0.1 \mu \mathrm{F}$

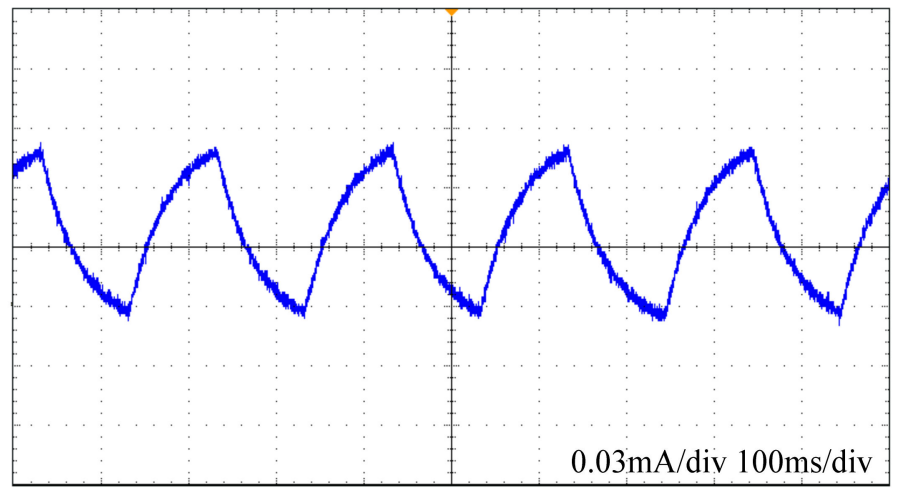

(b) $C_{Y}=0.2 \mu \mathrm{F}$

Figure 10. Cont. 


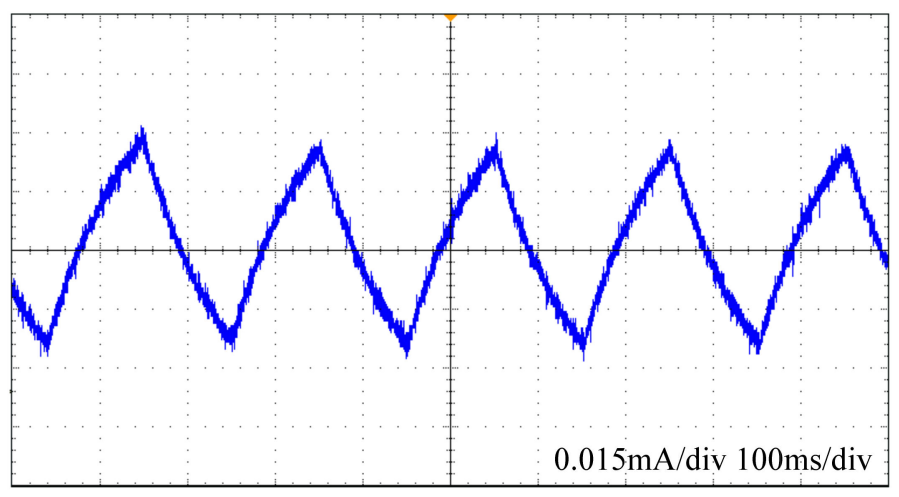

(c) $C_{\gamma}=0.4 \mu \mathrm{F}$

Figure 10. Waveform of grounding current with different values of $C_{Y}$. (a) $C_{Y}=0.1 \mu \mathrm{F}$. (b) $C_{Y}=0.2 \mu$. (c) $C_{Y}=0.4 \mu \mathrm{F}$.

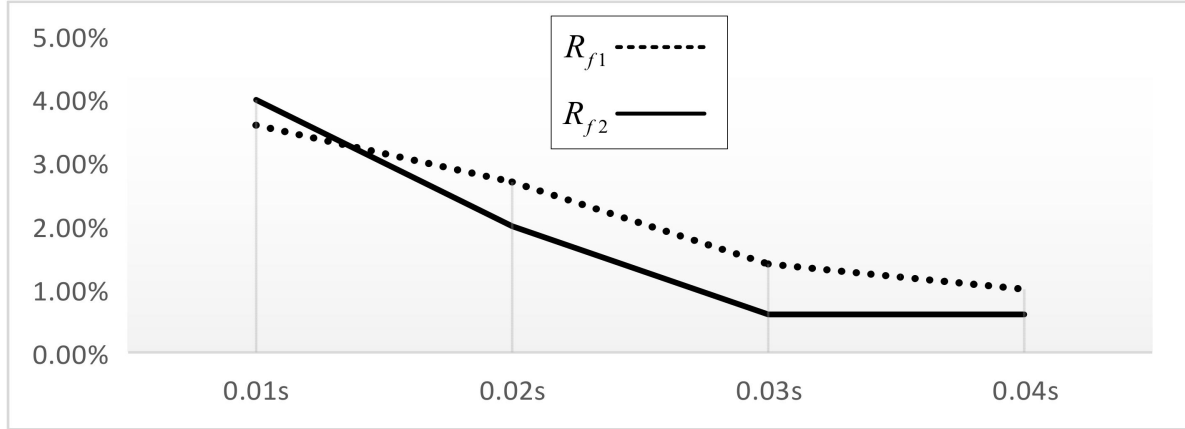

Figure 11. RE\% with different sampling intervals.

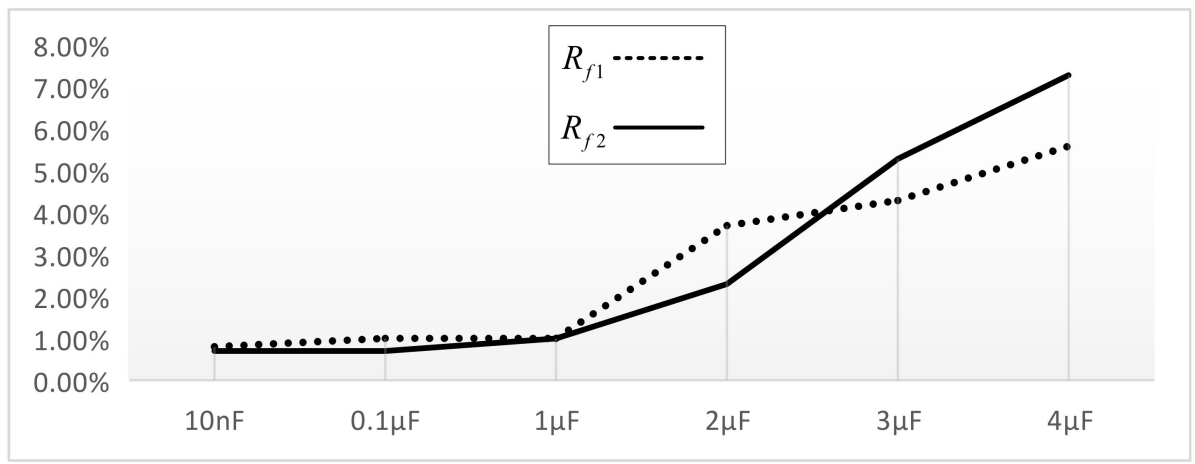

Figure 12. RE\% with different values of $C Y$.

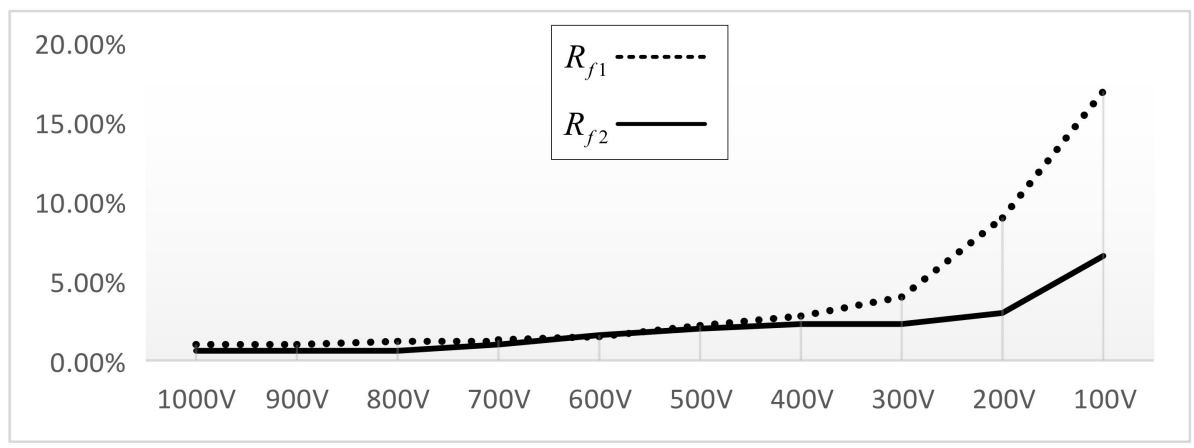

Figure 13. RE\% with different DC voltages. 
Table 2. Data results with different bridge resistors.

\begin{tabular}{cccccc}
\hline $\boldsymbol{R}_{\boldsymbol{a}}(\mathrm{k} \boldsymbol{\Omega})$ & $\boldsymbol{R}_{\boldsymbol{b}}(\mathrm{k} \boldsymbol{\Omega})$ & $\boldsymbol{R}_{\boldsymbol{f} \mathbf{1}}(\mathrm{k} \boldsymbol{\Omega})$ & $\mathbf{R E} \%$ of $\boldsymbol{R}_{\boldsymbol{f} \mathbf{1}}$ & $\boldsymbol{R}_{\boldsymbol{f} \mathbf{2}}(\mathbf{k} \boldsymbol{\Omega})$ & $\mathbf{R E} \%$ of $\boldsymbol{R}_{\boldsymbol{f} \mathbf{2}}$ \\
\hline 1000 & 200 & 1010 & $1.0 \%$ & 302 & $0.6 \%$ \\
800 & 250 & 1014 & $1.4 \%$ & 305 & $1.6 \%$ \\
600 & 300 & 1020 & $2.0 \%$ & 309 & $3.0 \%$ \\
500 & 350 & 1045 & $4.5 \%$ & 316 & $5.3 \%$ \\
\hline
\end{tabular}

Table 3. Comparison of monitoring data in $v_{\mathrm{dc}}=800 \mathrm{~V}, R_{f 1}=1000 \mathrm{k} \Omega, R_{f 2}=300 \mathrm{k} \Omega$.

\begin{tabular}{ccccc}
\hline & \multicolumn{2}{c}{ Traditional Method } & \multicolumn{2}{c}{ Proposed Method } \\
\hline$C_{\boldsymbol{Y}}$ & Monitoring Time & RE\% & Monitoring Time & RE\% \\
\hline $10 \mathrm{nF}$ & $0.2 \mathrm{~s}$ & $0.8 \%$ & $0.2 \mathrm{~s}$ & $0.8 \%$ \\
$0.1 \mu \mathrm{F}$ & $0.3 \mathrm{~s}$ & $1.0 \%$ & $0.2 \mathrm{~s}$ & $1.2 \%$ \\
$1 \mu \mathrm{F}$ & $1.35 \mathrm{~s}$ & $2.4 \%$ & $0.2 \mathrm{~s}$ & $1.8 \%$ \\
$2 \mu \mathrm{F}$ & $2.51 \mathrm{~s}$ & $3.5 \%$ & $0.2 \mathrm{~s}$ & $3.7 \%$ \\
$3 \mu \mathrm{F}$ & $3.67 \mathrm{~s}$ & $5 \%$ & $0.2 \mathrm{~s}$ & $5.3 \%$ \\
$4 \mu \mathrm{F}$ & $4.83 \mathrm{~s}$ & $6.6 \%$ & $0.2 \mathrm{~s}$ & $7.3 \%$ \\
\hline
\end{tabular}

Table 4. Comparison of monitoring data in $v_{\mathrm{dc}}=400 \mathrm{~V}, R_{f 1}=1000 \mathrm{k} \Omega, R_{f 2}=300 \mathrm{k} \Omega$.

\begin{tabular}{ccccc}
\hline & \multicolumn{2}{c}{ Traditional Method } & \multicolumn{2}{c}{ Proposed Method } \\
\hline$C_{\boldsymbol{Y}}$ & Monitoring Time & $\mathbf{R E} \%$ & Monitoring Time & RE\% \\
\hline $10 \mathrm{nF}$ & $0.2 \mathrm{~s}$ & $1.3 \%$ & $0.2 \mathrm{~s}$ & $1.3 \%$ \\
$0.1 \mu \mathrm{F}$ & $0.29 \mathrm{~s}$ & $1.7 \%$ & $0.2 \mathrm{~s}$ & $2.8 \%$ \\
$1 \mu \mathrm{F}$ & $0.34 \mathrm{~s}$ & $3.2 \%$ & $0.2 \mathrm{~s}$ & $4.1 \%$ \\
$2 \mu \mathrm{F}$ & $2.49 \mathrm{~s}$ & $5.8 \%$ & $0.2 \mathrm{~s}$ & $6.2 \%$ \\
$3 \mu \mathrm{F}$ & $3.64 \mathrm{~s}$ & $8.5 \%$ & $0.2 \mathrm{~s}$ & $9 \%$ \\
$4 \mu \mathrm{F}$ & $4.79 \mathrm{~s}$ & $11 \%$ & $0.2 \mathrm{~s}$ & $12 \%$ \\
\hline
\end{tabular}

Table 5. Comparison of monitoring data in $v_{\mathrm{dc}}=800 \mathrm{~V}, R_{f 1}=100 \mathrm{k} \Omega, R_{f 2}=100 \mathrm{k} \Omega$.

\begin{tabular}{ccccc}
\hline & \multicolumn{2}{c}{ Traditional Method } & \multicolumn{2}{c}{ Proposed Method } \\
\hline$C_{Y}$ & Monitoring Time & RE\% & Monitoring Time & RE\% \\
\hline $10 \mathrm{nF}$ & $0.2 \mathrm{~s}$ & $0.7 \%$ & $0.2 \mathrm{~s}$ & $0.7 \%$ \\
$0.1 \mu \mathrm{F}$ & $0.23 \mathrm{~s}$ & $1.0 \%$ & $0.2 \mathrm{~s}$ & $0.9 \%$ \\
$1 \mu \mathrm{F}$ & $0.65 \mathrm{~s}$ & $2.2 \%$ & $0.2 \mathrm{~s}$ & $1.6 \%$ \\
$2 \mu \mathrm{F}$ & $1.11 \mathrm{~s}$ & $3.4 \%$ & $0.2 \mathrm{~s}$ & $2.4 \%$ \\
$3 \mu \mathrm{F}$ & $1.57 \mathrm{~s}$ & $4.7 \%$ & $0.2 \mathrm{~s}$ & $4.1 \%$ \\
$4 \mu \mathrm{F}$ & $2 \mathrm{~s}$ & $6.3 \%$ & $0.2 \mathrm{~s}$ & $5.2 \%$ \\
\hline
\end{tabular}

\section{Conclusions}

For the DC-IM circuit of EVs, the traditional unbalanced electric bridge method switches the positive and negative bridge resistances and calculates the insulation resistance value by sampling the positive and negative bridge voltages. However, when the DC positive and negative poles have GC, the bridge voltages must be sampled after the capacitor is charged completely; thus, the measurement time is very long. This study proposes a novel method of DC-IM using a three-point climbing algorithm. The insulation resistance can be calculated by sampling the voltage of the positive and negative bridges three times and keeping the sampling interval equal. Moreover, the method filters and automatically corrects the three sampling voltages, which can improve the accuracy of the calculation results. Combined with experimental data, the following conclusions can be drawn: (1) The advantage of proposed method can perform a faster time and maintain a constant monitoring period compared with the traditional method. (2) The restriction of proposed method only apply in larger GC situation. 
If GC is small, the traditional method could be used. (3) The characteristics of proposed method: Increasing the sampling interval, increasing the difference between $R_{a}$ and $R_{b}$, increasing DC voltage $v_{\mathrm{dc}}$, all make the results more accurate. Overall, the proposed method can be applied to some practical industrial applications. The future work is to study how to set the constant $C_{a}$ to determine which method to use or find a different rule to generally judge the value of GC, and study a method to reduce error when $v_{\mathrm{dc}}$ is constant changing.

Author Contributions: J.D. and T.Q.Z. provided the method and solution; Y.Y. and H.Z. performed the experiments; J.D. and Y.Y. analyzed the data; J.D. and Y.Z. wrote the paper; T.Q.Z. and H.L. checked paper.

Funding: This research received no external funding.

Conflicts of Interest: The authors declare no conflict of interest.

\section{References}

1. Hou, Y.P.; Zhou, W.; Shen, C.Y. Experimental investigation of gas-tightness and electrical insulation of fuel cell stack under strengthened road vibrating conditions. Int. J. Hydrogen Energy 2011, 36, 13763-13768. [CrossRef]

2. Gyftakis, K.N.; Sumislawska, M.; Kavanagh, D.F.; Howey, D.A.; McCulloch, M.D. Dielectric Characteristics of Electric Vehicle Traction Motor Winding Insulation Under Thermal Aging. IEEE Trans. Ind. Appl. 2016, 52, 1398-1404.

3. Dannier, A. Overview of Main Electric Subsystems of Zero-Emission Vehicles. Propuls. Syst. 2019, 1-31. [CrossRef]

4. Vahedipour-Dahraie, M.; Rashidizaheh-Kermani, H.; Najafi, H.R.; Anvari-Moghaddam, A.; Guerrero, J.M. Coordination of EVs Participation for Load Frequency Control in Isolated Microgrids. Appl. Sci. 2017, 7, 539. [CrossRef]

5. Rafique, M.K.; Khan, S.U.; Zaman, M.S.U.; Mehmood, K.K.; Haider, Z.M.; Ali Bukhar, S.B.; Kim, C. An Intelligent Hybrid Energy Management System for a Smart House Considering Bidirectional Power Flow and Various EV Charging Techniques. Appl. Sci. 2019, 9, 1658. [CrossRef]

6. Kim, J.; Kim, H.; Cho, Y.; Kim, H.; Cho, J. Application of a DC Distribution System in Korea: A Case Study of the LVDC Project. Appl. Sci. 2019, 9, 1074. [CrossRef]

7. Holger Potdevin, D.-I. Insulation Monitoring in High Voltage Systems for Hybrid and Electric Vehicles. High Voltage Safety. ATZ Elektron. 2009, 4, 28-31. [CrossRef]

8. Pan, X.F.; Rinkleff, T.; Willmann, B.; Vick, R. PSpice simulation of surge testing for electrical vehicles. In Proceedings of the 2012 International Symposium on Electromagnetic Compatibility (EMC EUROPE), Rome, Italy, 17-21 September 2012.

9. Liu, Y.-C.; Lin, C.-Y. Insulation fault detection circuit for ungrounded DC power supply systems. In Proceedings of the 2012 IEEE Sensors, Taipei, Taiwan, 28-31 October 2012.

10. Zhao, C.Y.; Jia, X.F.; Hao, Z.F. The new method of monitoring DC system insulation on-line. In Proceedings of the 27th Annual Conference of the IEEE Industrial Electronics Society, IECON'01, Denver, CO, USA, 29 November-2 December 2001.

11. Jiang, J.S.; Ji, H. Study of Insulation Monitoring Device for DC System Based on Multi-switch Combination. In Proceedings of the Second International Symposium on Computational Intelligence and Design, ISCID'09, Changsha, China, 12-14 December 2009.

12. Li, J.X.; Wu, Z.Q.; Fan, Y.Q.; Wang, Y.Y.; Jiang, J.C. Research on Insulation Resistance On-Line Monitoring for Electric Vehicle. In Proceedings of the 2005 International Conference on Electrical Machines and Systems, Nanjing, China, 27-29 September 2005.

13. Wei, X.Y.; Bi, L.; Sun, Z.C. A method of insulation failure detection on electric vehicle based on FPGA. In Proceedings of the 2008 IEEE Vehicle Power and Propulsion Conference, VPPC'08, Harbin, China, 3-5 September 2008.

14. Zheng, M.X. The Design of Insulation Monitoring Device for Electric Vehicle's Battery Pack. Adv. Mater. Res. 2013, 718-720, 1422-1428. [CrossRef]

15. Bao, Y.; Jiang, J.C.; Zhang, W.G.; Wang, J.Y.; Wen, J.P. Novel On-line Insulation Supervising Method for DC System. High Volt. Eng. 2011, 37, 333-337. 
16. Wu, Z.J.; Wang, L.F. A novel insulation resistance monitoring device for Hybrid Electric Vehicle. In Proceedings of the 2008 IEEE Vehicle Power and Propulsion Conference, VPPC'08, Harbin, China, 3-5 September 2008.

17. Neti, P.; Grubic, S. Online broadband insulation spectroscopy of induction machines using signal injection. IEEE Trans. Ind. Appl. 2017, 53, 1054-1062. [CrossRef]

18. Chiang, Y.-H.; Sean, W.-Y.; Huang, C.-Y.; Chiang Hsieh, L.-H. Adaptive control for estimating insulation resistance of high-voltage battery system in electric vehicles. Environ. Prog. Sustain. Energy 2017, 36, 1882-1887. [CrossRef]

19. Li, D.H.; Jia, W. Application of Chaos Theory in Grounaing Fault Detection of DC Power Supply System. High Volt. Eng. 2005, 31, 79-82.

20. Li, D.H.; Shi, L.T. An Overall Scheme for Ground Fault Detection in DC Systems of Power Plants and Substations. Power Syst. Technol. 2005, 29, 56-59.

21. Song, C.X.; Shao, Y.L.; Song, S.X.; Peng, S.L.; Zhou, F.; Chang, C.; Wang, D. Insulation Resistance Monitoring Algorithm for Battery Pack in Electric Vehicle Based on Extended Kalman Filtering. Energies 2017, $10,714$. [CrossRef]

(C) 2019 by the authors. Licensee MDPI, Basel, Switzerland. This article is an open access article distributed under the terms and conditions of the Creative Commons Attribution (CC BY) license (http://creativecommons.org/licenses/by/4.0/). 